\section{PREVALENCE OF EXTRA-CARDIAC FINDINGS DETECTED BY CARDIAC MRI IN INHERITED VS ACQUIRED CARDIOVASCULAR DISEASES}

${ }^{1,2, *} \mathrm{AM}$ Amadu, ${ }^{1} \mathrm{~A}$ Baritussio, ${ }^{1} \mathrm{~A}$ Ghosh Dastidar, ${ }^{1} \mathrm{JCL}$ Rodrigues, ${ }^{2} \mathrm{P}$ Crivelli, ${ }^{2} \mathrm{~GB}$ Meloni, ${ }^{2} \mathrm{M}$ Conti, ${ }^{1} \mathrm{C}$ Bucciarelli-Ducci. ${ }^{1}$ Bristol Heart Institute, Bristol NIHR Cardiovascular Biomedical Research Unit (BRU), Bristol, UK; ${ }^{2}$ Department of Surgical, Microsurgical and Medical Sciences, Institute of Radiological Sciences, University of Sassari, Sassari, Italy

\subsection{6/heartjnl-2016-309668.1}

Introduction With its large field of view, Cardiovascular Magnetic Resonance (CMR) allows the detection of extra-cardiac pathologies (ECP). Both cardiologists and radiologists should be able to recognise ECP and identify those requiring further investigation. The aim of our study is to assess the difference in prevalence of ECP in patients with suspected inherited cardiac conditions vs acquired heart disease.

Materials and methods We reviewed 1.817 consecutive clinical CMR studies performed in the biggest CMR department in Southwest England to look for ECP. Demographic characteristics and scans indications were also recorded. For each scan the presence of ECP and its relevance (need for further investigation, i.e. suspected lung malignancy) was assessed. The internal record system (Picture Achievement and Communication System, PACS) was used to check whether the ECP were previously known, or whether it represents a new finding.

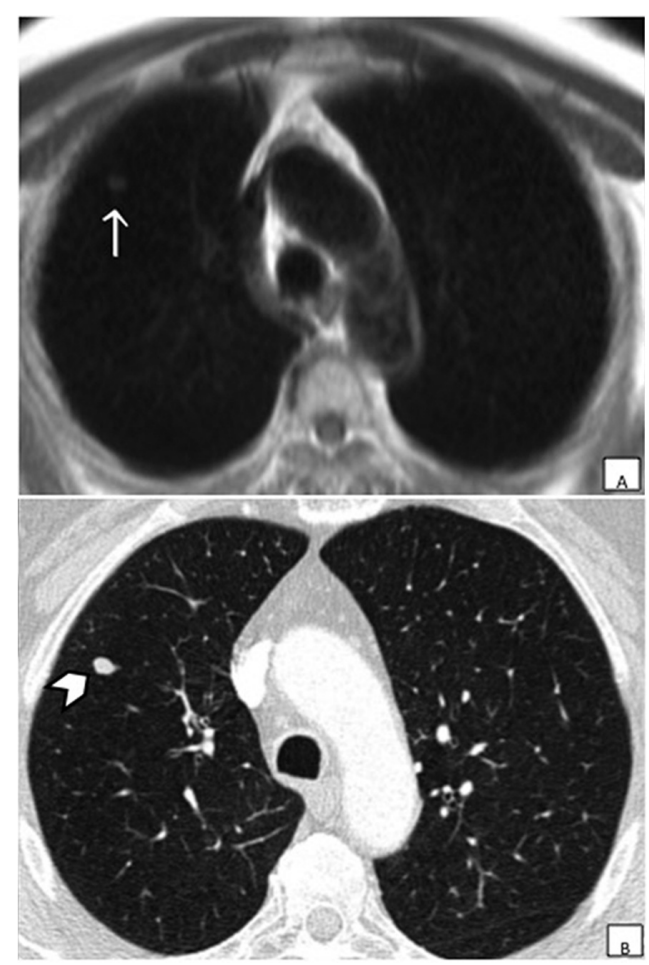

Abstract 1 Figure 1 (A) Axial Haste showed a nodule (arrow) in the superior lobe of the right lung. (B) On the High Resolution Computed Tomography (HRCT), performed to further assess the ECP, the presence of the nodule in the superior lobe of the right lung was confirmed (head-arrow)

Results We analysed 1,817 scans, referred for the assessment of inherited cardiac condition (Group $\mathrm{A}, \mathrm{n}=906$ ) and acquired heart disease (Group B, $\mathrm{n}=911$ ). There was no significant difference in prevalence of ECP between the two groups ( $p=$
0.63). ECP were found in $26 \%$ of patient in Group A, $4 \%$ of which requiring further assessment; 69\% previously unknown (Figure 1). ECP were reported in $27 \%$ of patients in Group B, $5 \%$ requiring further assessment; $68 \%$ were previously unknown.

Conclusion One in four patient has an extra-cardiac finding and the prevalence of ECP did not differ in patients presenting with inherited conditions vs acquired heart disease.

\section{CLINICAL APPLICATION OF CARDIOVASCULAR MAGNETIC RESONANCE IN PATIENTS WITH MR- CONDITIONAL DEVICES: SAFETY, FEASIBILITY AND CLINICAL IMPACT}

*A Baritussio, E De Garate, A Ghosh Dastidar, N Ahmed, A Scatteia, J Rodrigues, C Lawton, A Nisbet, E Duncan, T Cripps, I Diab, G Thomas, C Bucciarelli-Ducci. Bristol Heart Institute, Bristol NIHR Cardiovascular Biomedical Research Unit (BRU), Bristol, UK

\subsection{6/heartjnl-2016-309668.2}

Background Implanted cardiac devices were previously considered unsuitable for CMR. With the development of MR-conditional devices, access to CMR has increased, despite concerns regarding image quality and diagnostic accuracy. We aimed to assess the clinical application of CMR in patients wearing MRconditional devices.

Materials and methods We retrospectively enrolled patients wearing MR-conditional devices undergoing a comprehensive CMR protocol (cine, early and late gadolinium enhancement, LGE) in a $1.5 \mathrm{~T}$ scanner (June 2012-November 2015). Every sequence was analysed by two independent observers and scored according to the effect of artefacts on image quality and interpretation (no, minor and major artefacts). Inter-observer agreement was assessed per sequence and as overall judgement on scan quality and interpretation. Clinical impact of CMR was defined as a change in diagnosis and in management. All devices were interrogated before and after CMR.

Abstract 2 Table 1 Cohen's kappa for inter-observer agreement on image quality and interpretation per sequence and as overall judgement

\begin{tabular}{lll}
\hline & Cohen's kappa & $\mathbf{p}$ \\
\hline HASTE & 0.378 & 0.001 \\
Long Axis Cine & 0.356 & 0.001 \\
Short Axis Cine & 0.532 & $<0.001$ \\
EGE & 0.398 & 0.003 \\
Long Axis LGE & 0.284 & 0.005 \\
Short Axis LGE & 0.516 & $<0.001$ \\
Overall Judgement & 0.454 & $<0.001$
\end{tabular}

EGE, early gadolinium enhancement; LGE, late gadolinium enhancement.

Results We enrolled 46 consecutive patients (28 male, mean age $56 \pm 16$ years) wearing MR-conditional pacemaker $(22,48 \%)$ and implantable loop recorder $(24,52 \%)$. All CMR scans were successfully completed and diagnostic: minor artefacts were recorded in 17 scans (37\%), major artefacts in 7 (15\%), and no artefacts in 22 (48\%). Additional FLASH sequences were performed in 9 patients (20\%) to overcome artefacts. Inter-observer agreement on image quality and interpretation was moderate, both overall (kappa 0.454, p < 0.0001) and per sequence, with the exception of long-axis LGE sequences, for which it was fair 


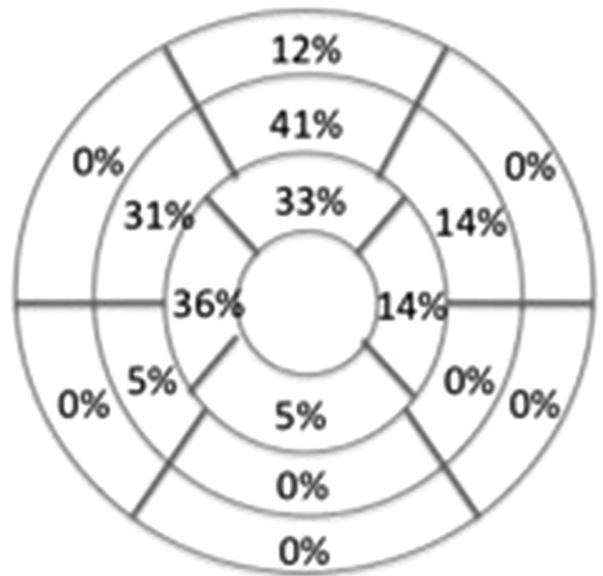

Cine sequences

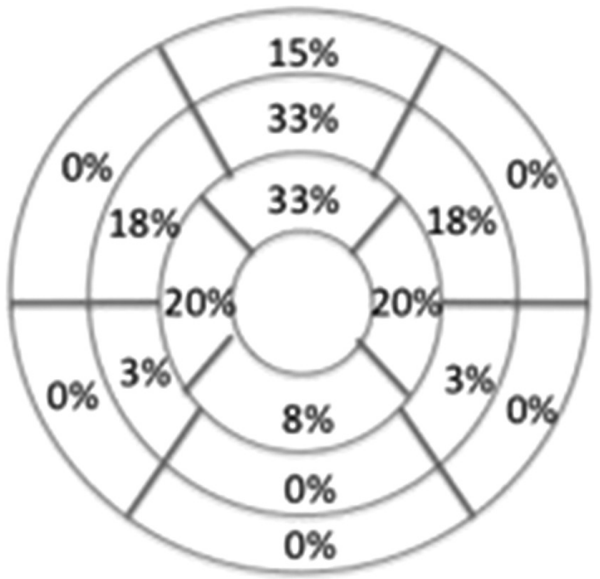

Post-contrast sequences

Abstract 2 Figure 1 Left ventricular segmental analysis to assess artefacts interference. Sixteen-segment model showing that artefacts mostly affect the mid-apical left ventricular anterior and anteroseptal walls, on cine and post-contrast sequences, respectively

(kappa 0.284, $\mathrm{p}=0.005$ ) (Table 1). Cine sequences were most affected by artefacts, mainly in the mid-apical left ventricular anterior wall and anteroseptum (Figure 1). No change in device parameters was reported after the scan. CMR had a clinical impact in 26 patients (57\%), determining a change in diagnosis in $16(35 \%)$, in management in $5(11 \%)$ and a change in both in 5 patients $(11 \%)$.

Conclusion With dedicated protocols and under strict monitoring of cardiac devices, CMR is safe and feasible in patients wearing MR-conditional devices, and it also has major clinical impact.

\section{CLINICAL UTILITY OF CARDIAC MRI IN YOUNG-MIDDLE AGED PATIENTS WITH HIGH-GRADE ATRIO- VENTRICULAR BLOCK}

${ }^{*}$ A Baritussio, A Ghosh Dastidar, N Ahmed, J Rodrigues, A Frontera, C Lawton, D Augustine, E McAlindon, C Bucciarelli-Ducci. NIHR Cardiovascular Biomedical Research Unit, Bristol Heart Institute, Bristol, UK

\subsection{6/heartjnl-2016-309668.3}

Background Atrio-ventricular (AV) block is a rare event in young-middle aged adults, often leading to pacemaker implantation without further investigation. We sought to assess the clinical utility of CMR in young-middle aged adults with high-grade AV block.

Methods We retrospectively analysed the CMR registry to collect data on consecutive high-grade AV block patients (18-60yrs) referred for CMR (September 2012-November 2015). Highgrade AVB was defined as Mobitz II $2^{\text {nd }}$ degree or complete AVB. All patients underwent a transthoracic echocardiogram (TTE) and a comprehensive CMR protocol (cine and late gadolinium enhancement, LGE). A change in diagnosis was defined as a new diagnosis compared to a multi-parametric pre-CMR diagnosis (based on clinical, ECG and TTE data).

Results We identified 34 patients (20 male, mean age $44 \pm 12$ years); 12 patients (34\%) had II degree AVB and 22 (66\%) complete AVB. Patients were referred to CMR for suspected ischaemic heart disease (IHD) in 4 patients (11\%) and non ischaemic heart disease (NIHD) in $24(71 \%)$; in 6 patients $(18 \%)$ preCMR diagnosis was unclear. CMR showed IHD in 3 patients (9\%) and NIHD in 11 patients (32\%); a structurally normal heart was found in 18 patients (53\%) and non-specific findings in $2(6 \%)$ (Table 1) (Figure 1). LGE was found in 12 patients (34\%), with predominant mid-wall pattern (58\%). There was

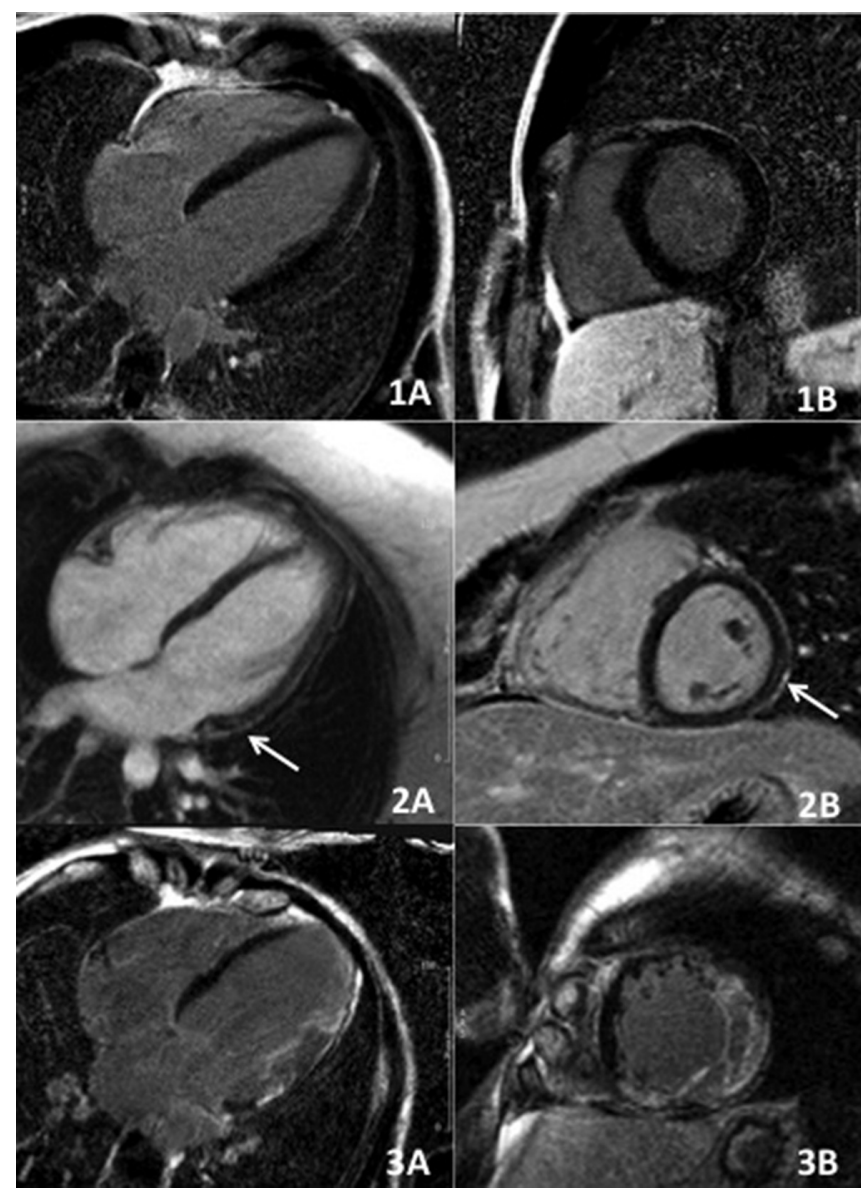

Abstract 3 Figure 1 CMR findings. Post-contrast four chamber longaxis $(1 \mathrm{~A})$ and short-axis (1B) view showing structurally normal heart. Post-contrast four chamber long-axis (2A) and short-axis (2B) view showing epicardial LGE in the basal to mid-cavity lateral wall (white arrow) in a patient with myocarditis. Post-contrast four chamber longaxis (3A) and short-axis (3B) view showing transmural myocardial LGE in the basal to apical lateral wall in a patient with left ventricular non compaction 